\title{
Evaluation of Nutritional Status of Cancer Patients during Treatment by Patient-Generated Subjective Global Assessment: a Hospital-Based Study
}

\author{
Dibyendu Sharma ${ }^{1 *}$, Ravi Kannan ${ }^{2}$, Ritesh Tapkire ${ }^{2}$, Soumitra Nath ${ }^{3}$
}

\begin{abstract}
Cancer patients frequently experience malnutrition. Cancer and cancer therapy effects nutritional status through alterations in the metabolic system and reduction in food intake. In the present study, fifty seven cancer patients were selected as subjects from the oncology ward of Cachar Cancer Hospital and Research Centre, Silchar, India. Evaluation of nutritional status of cancer patients during treatment was carried out by scored Patient-Generated Subjective Global Assessment (PG-SGA). The findings of PG-SGA showed that 15.8\% (9) were well nourished, $31.6 \%$ (18) were moderately or suspected of being malnourished and $52.6 \%(30)$ were severely malnourished. The prevalence of malnutrition was highest in lip/oral $(33.33 \%)$ cancer patients. The study showed that the prevalence of malnutrition $(\mathbf{8 4 . 2 \%})$ was high in cancer patients during treatment.
\end{abstract}

Keywords: Nutritional status - cancer - scored patient - generated subjective global assessment - malnourished

Asian Pac J Cancer Prev, 16 (18), 8173-8176

\section{Introduction}

Nutrition is an important factor in the treatment and progression of cancer. The majority of cancer patients experience weight loss as their disease progresses and, in general, weight loss is a major prognostic indicator of poor survival and impaired response to cancer treatment (Khoshnevis et al., 2012). Cancer patients are particularly susceptible to nutritional depletion due to the combined effects of the malignant disease and its treatment (Amaral et al., 2008; Paccagnella et al., 2010).

With increasing incidence of cancer, identification and management of nutritional deficiencies are needed. According to guidelines from the European Society of Parenteral and Enteral Nutrition (ESPEN) and American Society of Parenteral and Enteral Nutrition (ASPEN), preoperative nutritional support should be considered in cancer patients. Further, for severely malnourished patients, they recommend performing surgery after administering preoperative nutritional support for more than 7 days (Weimann et al., 2006; Braga et al., 2009). Studies indicate that malnutrition and weight loss are common among 20 to $97 \%$ of oncologic patients (Kern and Norton, 1988; Ollenschlager et al., 1991; Abe et al., 2013; Gabrielson et al., 2013; Malihi et al., 2013) and the prevalence of malnutrition depends on the tumour type, location, stage and treatment (Shike, 1996).

The consequences of malnutrition may include an increased risk of complications, decreased response and tolerance to treatment, impaired quality of life and decreased survival rate (Ottery, 1996; Nitenberg and Raynard, 2000). Factors affecting a person's food intake, such as difficulties swallowing and loss of appetite play an important role in quality of life (Hickson and Frost, 2004).

The prevalence and magnitude of a diminished nutritional status varies with individual treatment regimens, it is widely accepted that the principal causes related to therapy are the result of commonly experienced side effects such as nausea, vomiting, anorexia, lethargy, diarrhoea, esophagitis, and dysphasia (Kyle et al., 2005; Odelli et al., 2005). The cancer burden continues to increase due to adoption of lifestyles and behaviours that increase the risk of getting cancer and the increase in population causing strait resources pushing the economies to produce more that causes pollution and also increases exposure of masses to carcinogens (Jemal et al., 2011). Cancer can alter metabolism of nutrients, thus leading to development of symptoms and disturbances of the Gastro Intestinal Tract (GIT) leading to malnutrition (Nitenberg and Raynard, 2000). Hence, having the right knowledge is vital to enable cope with the symptoms as the treatment goes on and even after treatment to prevent relapse.

\section{Materials and Methods}

\section{Study design}

Present study was an observational study in which the standard questionnaire of PG-SGA was used to evaluate nutritional status of the patient. The participants enrolled in the study comprised of cancer patients who were receiving 
surgery, radiotherapy, chemotherapy or a combination of these or had completed treatment and was on follow-up care. A total of 57 patients were interviewed in the present study, age group between 28 to 75 years, comprising of 33 males and 24 females. All the gathered data were analysed by MS-Excel.

\section{Inclusion and exclusion criteria}

Patients who were diagnosed with cancer, aged at least 18 years, admitted to medical facility were eligible for inclusion in the study. The patients who were too ill to understand and respond were excluded.

\section{Results and Discussion}

Demographic characteristics of patients who were interviewed are described in Table 1.They had various forms of cancer. Out of the 57 subjects, $40.35 \%$ had cancer in lip/oral cavity followed by oesophageal cancer $(15.79 \%)$ and least being bone tumour $(1.75 \%)$. Distribution according to the site of cancer are given in Table 2..

Among the participants enrolled in the study, they were treated with radiotherapy chemotherapy or chemoradiotherapy. In most cases, patients had undergone surgery along with different therapy. Number of patients receiving different therapy and surgery are illustrated in Figure 1 .

BMI of participants were classified in different groups (Table 3) and the analysis showed that majority of them have imbalance weight-height ratio. It has also been observed that $80.70 \%$ of patients had less than usual foodintake, $15.79 \%$ of patients had unchanged food-intake and $3.51 \%$ of patients had more than usual food-intake over the preceding month (Table 4) and this may result in poor nutritional status and intolerance treatment. In a study (Sattianayagam et al., 2013) found that $55 \%$ of patients had less than usual food-intake, $45 \%$ of patients had unchanged food-intake and $4 \%$ of patients had more than usual food-intake over the preceding month.

The study tells us that the most common cause of food-intake reduction during the past two weeks were; no appetite $(38.60 \%)$, nausea $(43.86 \%)$, constipation $(15.79 \%)$, mouth sores $(17.54 \%)$ and pain $(8.77 \%)$ as seen in Table 4 . The problems most frequently endorsed by patients as contributing to psychological distress was depression $(73.88 \%)$ and the observation was found to be in correlation with other studies (Dyrbye et al., 2006; Ma et al., 2013).

Examining patient's weight loss revealed that 15 out of 57 patients $(26.32 \%$ ) did not lose weight and $19.30 \%$ had gained weight during the last one month of the study period. 5 patients were found to had weight loss of less than $3 \%, 7$ patients were found to had weight loss of 3-5 $\%, 13$ patients were found to had weight loss of 5-10\% and 6 patients were found to had weight loss of more than $10 \%$. Analysis of percentage of weight loss shows that $54.39 \%$ of the participants had weight loss of more than $3 \%$ in the last month or $5 \%$ over 6 months which is an early signs of malnutrition (Sattianayagam et al., 2013).

It has also been evidenced that only $1.75 \%$ of the subjects have normal activities with no limitations in their day-to-day activities over the past month. $17.54 \%$ of the subjects were able to do little activity and spend most of the day in bed or chair; $42.11 \%$ of the subjects have rate their activities as not their normal self, but able to be up and about with normal activities; $36.84 \%$ of the subjects have rate their activities as not felling up to most things, but in bed or chair less than half the day and $1.75 \%$ of the subjects were pretty much bedridden and rarely out of bed. The findings suggest that cancer patients both during and after treatments may be malnourished and have negative impact hence, on their day-to-day activities and function and treatment tolerance. So, proper planning and nutrition intervention before, during and after treatment is required.

Physical examination conducted for subjective

Table 1. Demographic Characteristics of Patients

\begin{tabular}{lrr}
\hline Characteristics & \multicolumn{2}{c}{ Number of patients } \\
\hline Age & $28-50$ years & $19(33.33 \%)$ \\
& $51-72$ years & $38(66.67 \%)$ \\
Religion & Hindu & $35(61.40 \%)$ \\
Muslim & $17(29.82 \%)$ \\
Income per Month & Christian & $5(8.80 \%)$ \\
(Indian Rupees) & $<5000$ & $37(64.91 \%)$ \\
Sanitation & $>5000$ & $20(35.09 \%)$ \\
& Squat toilet & $19(33.33 \%)$ \\
Food Habit & Pit toilet & $38(66.67 \%)$ \\
& Vegetarian & $1(1.75 \%)$ \\
Water Source & Non-Vegetarian & $56(98.25 \%)$ \\
& PHE Department & $31(54.40 \%)$ \\
Well & $8(14.03 \%)$ \\
Drinking Water Treatment & Tube-Well & $8(14.03 \%)$ \\
Tobacco Use & Pond & $10(17.54 \%)$ \\
Liquor & Boiling & $6(10.53 \%)$ \\
& Untreated & $33(57.90 \%)$ \\
& Chewing & $52(91.58 \%)$ \\
& Smoking & $31(54.38 \%)$ \\
& Alcohol & $8(14.03 \%)$ \\
\hline
\end{tabular}

Table 2. Distribution according to the site of Cancer

\begin{tabular}{lc}
\hline Cancer site & Number of patients \\
\hline Lip/oral cavity & $23(40.35 \%)$ \\
Pharynx & $3(5.26 \%)$ \\
Larynx & $2(3.51 \%)$ \\
Oesophagus & $9(15.79 \%)$ \\
Bone tumor & $1(1.75 \%)$ \\
Lymphoma & $2(3.51 \%)$ \\
Breast & $4(7.02 \%)$ \\
Lung & $4(7.02 \%)$ \\
Ovary & $3(5.26 \%)$ \\
Colon & $2(3.51 \%)$ \\
Rectum & $4(7.02 \%)$ \\
\hline
\end{tabular}

Table 3. BMI Classification of Participants

\begin{tabular}{lr}
\hline Categories & \multicolumn{1}{c}{ BMI } \\
\hline Very severely underweight $(<15)$ & $12(21.05 \%)$ \\
Severely underweight $(15-16)$ & $6(10.53 \%)$ \\
Underweight (16-18.5) & $14(24.56 \%)$ \\
Normal (18.5-25) & $21(36.84 \%)$ \\
Overweight (25-30) & $3(5.26 \%)$ \\
Obese class 1 (30-35) & $1(1.75 \%)$ \\
\hline
\end{tabular}


Evaluation of Nutritional Status of Cancer Patients during Treatment by PG-SGA: a Hospital Based Study

Table 4. Frequency of Variables Affecting Patients' Nutritional Status (PG-SGA Score)

\begin{tabular}{lr}
\hline Clinical characteristics & Number of Patients \\
\hline Food intake & \\
Unchanged & $(15.79 \%)$ \\
More than usual & $2(3.51 \%)$ \\
Less than usual & $46(80.70 \%)$ \\
Problem/Symptoms & \\
No appetite & $22(38.60 \%)$ \\
Nausea & $25(43.86 \%)$ \\
Constipation & $9(15.79 \%)$ \\
Mouth sores & $10(17.54 \%)$ \\
Pain & $5(8.77 \%)$ \\
Depression & $42(73.68 \%)$ \\
Weight loss & \\
$>10 \%$ & $6(10.53 \%)$ \\
5-10\% & $13(22.81 \%)$ \\
3-5\% & $7(12.28 \%)$ \\
$<3$ & $5(8.77 \%)$ \\
Weight increased & $11(19.30 \%)$ \\
Weight unchanged & $15(26.32 \%)$ \\
Physical activity & \\
Rarely out of bed & $1(1.75 \%)$ \\
Less than half the day ,in bed & $21(36.84 \%)$ \\
Fairly normal activities & $24(42.11 \%)$ \\
More than half the day , in bed/chair & $10(17.54 \%)$ \\
Normal no limitation & $1(1.75 \%)$ \\
Physical Examination & \\
Severe & $14(24.56 \%)$ \\
Moderate & $21(36.84 \%)$ \\
Mild & $11(19.30 \%)$ \\
Normal & $11(19.30 \%)$ \\
\hline & \\
\hline
\end{tabular}

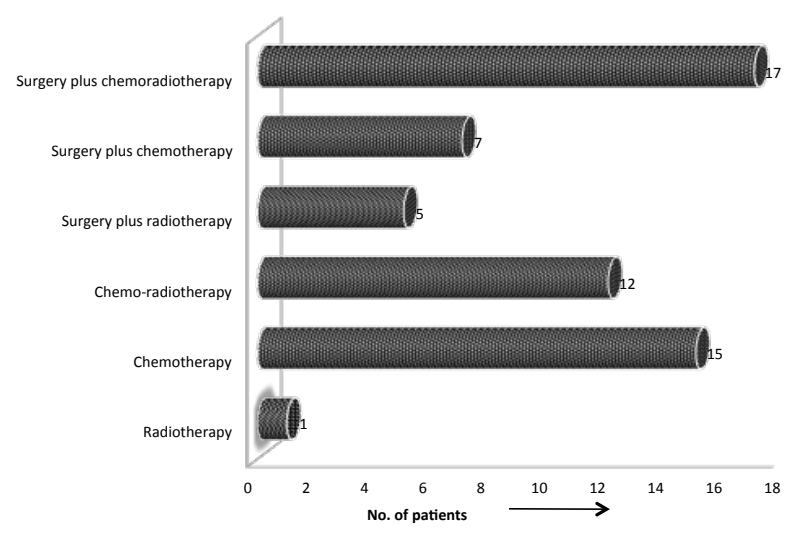

Figure 1. Different Type of Treatments and their Combinations



Figure 2. Frequency of Severity of Malnutrition (PGSGA score) evaluation of fat, muscle and fluid status showed that $24.56 \%$ of patients had severe deficit, $36.84 \%$ of patients had moderate deficit, $19.30 \%$ of patients had mild deficit and only $19.30 \%$ of patients had no deficit. Thus, indicating that the prevalence of malnutrition is very high among them during treatment.

According to PG-SGA score (Fig 2) $15.79 \%$ of patients were well-nourished or anabolic (SGA-A); $31.58 \%$ of patients had moderate or suspected malnutrition (SGA-B); $52.63 \%$ of patients were severely malnourished. Thus, the prevalence of malnutrition in the study population was high $(84.21 \%)$ and it depends on the tumour type, tumour location, stage of the disease, and treatment received and on the type of nutritional assessment method used (Shike, 1996; Bauer et al., 2002).

\section{Co-relation between $P G$-SGA score and BMI}

The prevalence of moderate to severe malnutrition in the patients was $84.21 \%$ (PG-SGA) and the prevalence of undernutrition was $56.14 \%$ (BMI). From the nutrition assessment tool of PG-SGA, the result we got indicated that BMI alone cannot be a reliable indicator of nutritional status of an individual in cancer patients. Previous studies in cancer patient groups also highlighted the limitations of using BMI as the sole measure of nutritional status (Desbrow et al., 2005; Isenring et al., 2006).

In conclusion, This observational study highlights the fact that nutritional issues are prevalent among cancer patients during treatment. High prevalence of malnutrition $(84.21 \%)$ was observed among cancer patients, and this was significantly associated with clinical symptoms directly related to the eating process. Nutritional screening is an important step needed to help intervene earlier in the Cancer patient's trajectory. Earlier detection of nutritional risk symptoms will result in thorough nutritional assessments and interventions that may help prevent further or pending malnutrition and weight loss during treatment and ultimately improve the quality of life of the advanced cancer patient.

\section{Acknowledgements}

The authors wish to extend their grateful thanks to Cachar Cancer Hospital and Research Centre, Silchar, Assam, India for providing the opportunity to carry out the present study. Authors also acknowledge the Hospital Society for funding the study.

\section{References}

Abe Vicente M, Barao K, Silva TD, et al (2013). What are the most effective methods for assessment of nutritional status in outpatients with gastric and colorectal cancer? Nutr Hosp, 28, 585-91.

Amaral TF, Antunes A, Cabral S, et al (2008). An evaluation of three nutritional screening tools in a Portuguese oncology centre. J Hum Nutr Diet, 21, 575-83.

Bauer J, Capra S, Ferguson M (2002). Use of the scored PatientGenerated Subjective Global Assessment (PG-SGA) as a nutrition assessment tool in patients with cancer. Eur J Clin Nutr, 56, 779-85.

Braga M, Ljungqvist O, Soeters P, et al (2009). ESPEN 
Guidelines on Parenteral Nutrition: surgery. Clin Nutr, 28, 378-86.

Desbrow B, Bauer J, Blum C, et al (2005). Assessment of nutritional status in hemodialysis patients using patientgenerated subjective global assessment. J Ren Nutr, 15, 211-6.

Dyrbye LN, Thomas MR, Shanafelt TD (2006). Systematic review of depression, anxiety, and other indicators of psychological distress among U.S. and Canadian medical students. Acad Med, 81, 354-73.

Gabrielson DK, Scaffidi D, Leung E, et al (2013). Use of an abridged scored Patient-Generated Subjective Global Assessment (abPG-SGA) as a nutritional screening tool for cancer patients in an outpatient setting. Nutr Cancer, 65, 234-9.

Hickson M, Frost G (2004). An investigation into the relationships between quality of life, nutritional status and physical function. Clin Nutr, 23, 213-21.

Isenring E, Cross G, Daniels L, et al (2006). Validity of the malnutrition screening tool as an effective predictor of nutritional risk in oncology outpatients receiving chemotherapy. Support Care Cancer, 14, 1152-6.

Jemal A, Bray F, Center MM, et al (2011). Global cancer statistics. CA Cancer J Clin, 61, 69-90.

Kern KA, Norton JA (1988). Cancer cachexia. JPEN J Parenter Enteral Nutr, 12, 286-98.

Khoshnevis N, Ahmadizar F, Alizadeh M, et al (2012). Nutritional assessment of cancer patients in Tehran, Iran. Asian Pac J Cancer Prev, 13, 1621-6.

Kyle UG, Genton L, Pichard C (2005). Hospital length of stay and nutritional status. Curr Opin Clin Nutr Metab Care, 8 , 397-402.

Ma L, Poulin P, Feldstain A, et al (2013). The association between malnutrition and psychological distress in patients with advanced head-and-neck cancer. Curr Oncol, 20, 554-60.

Malihi Z, Kandiah M, Chan YM, et al (2013). Nutritional status and quality of life in patients with acute leukaemia prior to and after induction chemotherapy in three hospitals in Tehran, Iran: a prospective study. J Hum Nutr Diet, 26, 123-31.

Nitenberg G, Raynard B (2000). Nutritional support of the cancer patient: issues and dilemmas. Crit Rev Oncol Hematol, 34, 137-68.

Odelli C, Burgess D, Bateman L, et al (2005). Nutrition support improves patient outcomes, treatment tolerance and admission characteristics in oesophageal cancer. Clin Oncol ( $R$ Coll Radiol), 17, 639-45.

Ollenschlager G, Viell B, Thomas W, et al (1991). Tumor anorexia: causes, assessment, treatment. Recent Results Cancer Res, 121, 249-59.

Ottery FD (1996). Definition of standardized nutritional assessment and interventional pathways in oncology. Nutrition, 12, 15-9.

Paccagnella A, Morello M, Da Mosto MC, et al (2010). Early nutritional intervention improves treatment tolerance and outcomes in head and neck cancer patients undergoing concurrent chemoradiotherapy. Support Care Cancer, 18, 837-45.

Sattianayagam PT, Lane T, Fox Z, et al (2013). A prospective study of nutritional status in immunoglobulin light chain amyloidosis. Haematologica, 98, 136-40.

Shike M (1996). Nutrition therapy for the cancer patient. Hematol Oncol Clin North Am, 10, 221-34.

Weimann A, Braga M, Harsanyi L, et al (2006). ESPEN guidelines on enteral nutrition: surgery including organ transplantation. Clin Nutr, 25, 224-44. 